\title{
Existence of positive solutions for eigenvalue problem of nonlinear fractional differential equations
}

\author{
Xiaoling $\operatorname{Han}^{*}$ and Hongliang Gao
}

\footnotetext{
* Correspondence: hanxiaoling@nwnu.edu.cn Department of Mathematics, Northwest Normal University, Lanzhou, 730070, P. R. China
}

\author{
Abstract \\ In this article, by using the fixed point theorem, existence of positive solutions for \\ eigenvalue problem of nonlinear fractional differential equations

$$
\left\{\begin{array}{l}
D_{0+}^{\alpha} u(t)+\lambda a(t) f(t, u(t))=0,0<t<1 \\
u(0)=u(1)=0
\end{array}\right.
$$ \\ is considered, where $1<\alpha<2$ is a real number, $D_{0_{+}}^{\alpha}$ is the standard Riemann- \\ Liouville derivate, $\lambda$ is a positive parameter and $a(t) \in C([0,1],[0, \infty)), f(t, u) \in C([0,1]$ \\ $\times[0, \infty),[0, \infty))$. \\ $\operatorname{MSC}(2010): 34 B 18$.
}

Keywords: fractional differential equation, positive solution, eigenvalue problem, fixed point

\section{Introduction}

Fractional differential equations have been of great interest recently. It is caused both by the intensive development of the theory of fractional calculus itself and by the applications of such constructions in various sciences such as physics, mechanics, chemistry, engineering, etc. For details, see [1-6] and references therein.

Recently, many results were obtained dealing with the existence and multiplicity of solutions of nonlinear fractional differential equations by the use of techniques of nonlinear analysis, see [7-22] and the reference therein. Bai and Lu [7] studied the existence of positive solutions of nonlinear fractional differential equation

$$
\left\{\begin{array}{l}
D_{0+}^{\alpha} u(t)+f(t, u(t))=0,0<t<1 \\
u(0)=u(1)=0
\end{array}\right.
$$

where $1<\alpha \leq 2$ is a real number, $D_{0_{+}}^{\alpha}$ is the standard Riemann-Liouville differentiation, and $f:[0,1] \times[0, \infty) \rightarrow[0, \infty)$ is continuous. They derived the corresponding Green function and obtained some properties as follows.

Proposition 1 The Green function $G(t, s)$ satisfies the following conditions:

(R1) $G(t, s) \in C([0,1] \times[0,1])$, and $G(t, s)>0$ for $t, s \in(0,1)$; 
(R2) There exists a positive function $\gamma \in C(0,1)$ such that

$$
\min _{\frac{1}{4} \leq t \leq \frac{3}{4}} G(t, s) \geq \gamma(s) \max _{0 \leq t \leq 1} G(t, s) \geq \gamma(s) G(s, s), s \in(0,1),
$$

where

$$
G(t, s)=\left\{\begin{array}{l}
\frac{[t(1-s)]^{\alpha-1}-(t-s)^{\alpha-1}}{\Gamma(\alpha)}, \quad 0 \leq s \leq t \leq 1 \\
\frac{[t(1-s)]^{-1}}{\Gamma(\alpha)}, \quad 0 \leq t \leq s \leq 1
\end{array}\right.
$$

It is well known that the cone plays a very important'”role in applying the Green function in research area. In [7], the authors cannot acquire a positive constant taken instead of the role of positive function $\gamma(s)$ with $1<\alpha<2$ in (1.2). In [9], Jiang and Yuan obtained some new properties of the Green function and established a new cone. The results can be stated as follows.

Proposition 2 The Green function $G(t, s)$ defined by (1.3) has the following properties: $G(t, s)=G(1-s, 1-t)$ and

$$
\frac{\alpha-1}{\Gamma(\alpha)} t^{\alpha-1}(1-t)(1-s)^{\alpha-1} s \leq G(t, s) \leq \frac{1}{\Gamma(\alpha)} t^{\alpha-1}(1-t)(1-s)^{\alpha-2} \quad \forall t, s \in(0,1) .
$$

Proposition 3 The function $G^{*}(t, s):=t^{2-\alpha} G(t, s)$ has the following properties:

$$
q(t) \Phi(s) \leq G^{*}(t, s) \leq \Phi(s) \quad \forall t, s \in[0,1] .
$$

where $q(t)=(\alpha-1) t(1-t), \Phi(s)=\frac{1}{\Gamma(\alpha)} s(1-s)^{\alpha-1}$.

The purpose of this article is to establish the existence of positive solutions for eigenvalue problem of nonlinear fractional differential equations

$$
\left\{\begin{array}{l}
D_{0+}^{\alpha} u(t)+\lambda a(t) f(t, u(t))=0, \quad 0<t<1 \\
u(0)=u(1)=0
\end{array}\right.
$$

where $1<\alpha<2$ is a real number, $D_{0_{+}}^{\alpha}$ is the standard Riemann-Liouville derivate, $\lambda$ is a positive parameter and $a(t) \in C([0,1],[0, \infty)), f(t, u) \in C([0,1] \times[0, \infty),[0, \infty))$.

\section{The preliminary lemmas}

For the convenience of the reader, we present here the necessary definitions from fractional calculus theory. These definitions can be found in the recent literature.

Definition 2.1 The fractional integral of order $\alpha>0$ of a function $y:(0, \infty) \rightarrow R$ is given by

$$
I_{0+}^{\alpha} \gamma(t)=\frac{1}{\Gamma(\alpha)} \int_{0}^{t}(t-s)^{\alpha-1} \gamma(s) d s
$$

provided the right side is pointwise defined on $(0, \infty)$.

Definition 2.2 The fractional derivative of order $\alpha>0$ of a function $y:(0, \infty) \rightarrow R$ is given by

$$
D_{0+}^{\alpha} \gamma(t)=\frac{1}{\Gamma(n-\alpha)}\left(\frac{d}{d t}\right)^{n} \int_{0}^{t} \frac{\gamma(s)}{(t-s)^{\alpha-n+1}} d s
$$

where $n=[\alpha]+1$, provided the right side is pointwise defined on $(0, \infty)$. 
Lemma 2.1 Let $\alpha>0$. If we assume $u \in C(0,1) \cap L(0,1)$, then the fractional differential equation

$$
D_{0+}^{\alpha} u(t)=0
$$

has $u(t)=C_{1} t^{\alpha-1}+C_{2} t^{\alpha-2}+\ldots+C_{N} t^{\alpha-N}, C_{i} \in R, i=1,2, \ldots, N$, where $N$ is the smallest integer greater than or equal to $\alpha$, as unique solutions.

Lemma 2.2 [7] Assume that $u L C(0,1) \cap L(0,1)$ with a fractional derivative of order $\alpha>0$ that belongs to $u \in C(0,1) \cap L(0,1)$. Then

$$
I_{0+}^{\alpha} D_{0+}^{\alpha} u(t)=u(t)+C_{1} t^{\alpha-1}+C_{2} t^{\alpha-2}+\cdots+C_{N} t^{\alpha-N}
$$

for some $C_{i} \in R, i=1,2, \ldots, N$.

Lemma 2.3 [7] Given $y \in C[0,1]$ and $1<\alpha \leq 2$, the unique solution of

$$
\left\{\begin{array}{l}
D_{0+}^{\alpha} u(t)+y(t)=0, \quad 0<t<1 \\
u(0)=u(1)=0
\end{array}\right.
$$

is

$$
u(t)=\int_{0}^{1} G(t, s) y(s) d s,
$$

where

$$
G(t, s)=\left\{\begin{array}{l}
\frac{[t(1-s)]^{\alpha-1}-(t-s)^{\alpha-1}}{\Gamma(\alpha)}, \quad 0 \leq s \leq t \leq 1, \\
\frac{[t(1-s)]^{\alpha-1}}{\Gamma(\alpha)}, \quad 0 \leq t \leq s \leq 1 .
\end{array}\right.
$$

Lemma 2.4 [10] Let $K$ be a cone in Banach space $E$. Suppose that $T: \bar{K}_{r} \rightarrow K$ is a completely continuous operator.

(i) If there exists $u_{0} \in K \backslash\{\theta\}$ such that $u-T u \neq \mu u_{0}$ for any $u \in \partial K_{r}$ and $\mu \geq 0$, then $i\left(T, K_{r}, K\right)=0$.

(ii) If $T u \neq \mu u$ for any $u \in \partial K_{r}$ and $\mu \geq 1$, then $i\left(T, K_{r}, K\right)=1$.

Lemma 2.5 [8] Let $P$ be a cone in Banach space $X$. Suppose that $T: P \rightarrow P$ is a completely continuous operator. If there exists a bounded open set $\Omega(P)$ such that each solution of

$$
u=\sigma T u, \quad u \in P, \sigma \in[0,1]
$$

satisfies $u \in \Omega(P)$, then the fixed point index $i(T, \Omega(P), P)=1$.

\section{The main results}

Let $E=C[0,1]$ be endowed with the ordering $u \leq v$ if $u(t) \leq v(t)$ for all $t \in[0,1]$, and the maximum norm, $\|u\|=\max _{0 \leq t \leq 1}|u(t)|$. Define the cone $P \subset E$ by $P=\{u \in E \mid u(t) \geq 0\}$, and

$$
K=\{u \in P|u(t) \geq q(t) \| u| \mid\} .
$$

where $q(t)$ is defined by (1.5).

It is easy to see that $P$ and $K$ are cones in $E$. For any $0<r<R<+\infty$, let $K_{r}=\{u \in K \mid$ $\|u\|<r\}, \quad \partial K_{r}=\{u \in K \mid \quad\|u\|=r\}, \quad \bar{K}_{r}=\{u \in K \mid\|u\| \leq r\}$ and $\bar{K}_{R} \backslash K_{r}=\{u \in K \mid r \leq\|u\| \leq R\}$. 
For convenience, we introduce the following notations

$$
\begin{gathered}
g_{0}=\lim _{u \rightarrow 0^{+}} \frac{g(u)}{u}, \quad g_{\infty}=\lim _{u \rightarrow+\infty} \frac{g(u)}{u} \\
\int_{\frac{1}{4}}^{\frac{3}{4}} G^{*}(\tau, s) a(s) q_{1}(s) d s=\max _{\frac{1}{4} \leq t \leq \frac{3}{4}} \int_{\frac{1}{4}}^{\frac{3}{4}} G^{*}(t, s) a(s) q_{1}(s) d s .
\end{gathered}
$$

We assume the following conditions hold throughout the article:

(H1) $a(t) \in C([0,1],[0, \infty)), a(t) \otimes 0$;

$(\mathrm{H} 2) f(t, u) \in C([0,1] \times[0, \infty),[0, \infty))$, and there exist $g \in C([0,+\infty),[0,+\infty)), q_{1}, q_{2}$ $\in C((0,1),(0,+\infty))$ such that

$$
q_{1}(t) g(u) \leq f\left(t, t^{\alpha-2} u\right) \leq q_{2}(t) g(u), \quad t \in(0,1), u \in[0,+\infty),
$$

where $\int_{0}^{1} q_{i}(s) d s<+\infty, i=1,2$.

By similar arguments to Lemma 4.1 and Theorem 1.3 of [9], we obtain the following result.

Lemma 3.1 Assume that $(\mathrm{H} 1)(\mathrm{H} 2)$ hold. Let

$$
T u(t):=\lambda \int_{0}^{1} G^{*}(t, s) a(s) f\left(s, s^{\alpha-2} u(s)\right) d s,
$$

then $T: K \rightarrow K$ is completely continuous. Moreover, if $u$ is a fixed point of $T$ in $\bar{K}_{R} \backslash K_{r}$, then $y=t^{\alpha-2} u$ is a positive solution of BVP (1.6).

By similar arguments to Theorems 2 and 3 of [18], we obtain the following result.

Theorem 3.1 Assume that $(\mathrm{H} 1)(\mathrm{H} 2)$ hold. Then, for each $\lambda$ satisfying

$$
\frac{16}{\left[(\alpha-1) \int_{\frac{1}{4}}^{\frac{3}{4}} G^{*}(\tau, s) a(s) q_{1}(s) d s\right] g_{\infty}}<\lambda<\frac{1}{\left(\int_{0}^{1} \Phi(s) a(s) q_{2}(s) d s\right) g_{0}}
$$

there exists at least one positive solution of BVP (1.6) in $K$.

Theorem 3.2 Assume that $(\mathrm{H} 1)(\mathrm{H} 2)$ hold. Then, for each $\lambda$ satisfying

$$
\frac{16}{\left[(\alpha-1) \int_{\frac{1}{4}}^{\frac{3}{4}} G^{*}(\tau, s) a(s) q_{1}(s) d s\right] g_{0}}<\lambda<\frac{1}{\left(\int_{0}^{1} \Phi(s) a(s) q_{2}(s) d s\right) g_{\infty}}
$$

there exists at least one positive solution of BVP (1.6) in $K$.

Set

$$
\begin{aligned}
& L_{1} u(t):=\int_{0}^{1} G^{*}(t, s) a(s) q_{1}(s) u(s) d s \\
& L_{2} u(t):=\int_{0}^{1} G^{*}(t, s) a(s) q_{2}(s) u(s) d s .
\end{aligned}
$$

It is clear that $L_{1}, L_{2}$ is a completely continuous linear operator and $L_{1}(P) \subset K, L_{2}(P)$ $\subset K$. By virtue of the Krein-Rutman theorem and Proposition 3, we have the following lemma.

Lemma 3.2 Assume that $(\mathrm{H} 1)(\mathrm{H} 2)$ hold. Then the spectral radius $r\left(L_{1}\right)>0, r\left(L_{2}\right)>0$ and $L_{1}, L_{2}$, respectively, has a positive eigenfunction $\phi_{1}, \phi_{2}$ corresponding to its first eigenvalue $\lambda_{1}=\left(r\left(L_{1}\right)\right)^{-1}, \lambda_{2}=\left(r\left(L_{1}\right)\right)^{-1}$, that is $\phi_{1}=\lambda_{1} L_{1} \phi_{1}, \phi_{2}=\lambda_{2} L_{2} \phi_{2}$. 
Obviously, $L_{1} \phi_{1} \in K, L_{2} \phi_{2} \in K$, so $\phi_{1} \in K, \phi_{2} \in K$.

In the following, we will obtain some existence results under some conditions concerning the first eigenvalue with respect to linear operator $L_{1}, L_{2}$.

Assume that

(H3) $\frac{\lambda_{1}}{g_{0}}<\lambda<\frac{\lambda_{2}}{g_{\infty}}$;

(H3) $\frac{\lambda_{1}}{g_{\infty}}<\lambda<\frac{\lambda_{2}}{g_{0}}$.

Theorem 3.3 Assume $(\mathrm{H} 1)(\mathrm{H} 2)(\mathrm{H} 3)$ hold. Then the BVP (1.6) has at least one positive solution.

Proof. First, by Lemma 3.1, we know that $T: K \rightarrow K$ is completely continuous.

By (H3), we have $g_{0}>\frac{\lambda_{1}}{\lambda}$, there exists $r_{1}>0$ such that

$$
g(u) \geq \frac{\lambda_{1}}{\lambda} u, \quad 0<u \leq r_{1} .
$$

Then for $u \in \partial K_{r_{1}}$, we have

$$
\begin{aligned}
(\mathrm{Tu})(t) & =\lambda \int_{0}^{1} G^{*}(t, s) a(s) f\left(s, s^{\alpha-2} u(s)\right) d s \\
& \geq \lambda \int_{0}^{1} G^{*}(t, s) a(s) q_{1}(s) g(u(s)) d s \\
& \geq \lambda_{1} \int_{0}^{1} G^{*}(t, s) a(s) q_{1}(s) u(s) d s=\lambda_{1}\left(L_{1} u\right)(t) .
\end{aligned}
$$

By Lemma 3.2, $\phi_{1}=\lambda_{1} L_{1} \phi_{1}$. We may suppose that $T$ has no fixed points on $\partial K_{r_{1}}$ (otherwise, the proof is finished). Now we show that

$$
u-T u \neq \mu \varphi_{1}, \quad u \in \partial K_{r 1}, \quad \mu \geq 0 .
$$

Assume by contradicts that there exist $u \in \partial K_{r_{1}}$ and $\mu_{1} \geq 0$ such that $u_{1}-T u_{1}=$ $\mu_{1} \phi_{1}$, then $\mu_{1}>0$ and $u_{1}=T u_{1}+\mu_{1} \phi_{1} \geq \mu_{1} \phi_{1}$. Let $\bar{\mu}=\sup \left\{\mu \mid u_{1} \geq \mu \varphi_{1}\right\}$, then $\bar{\mu} \geq \mu_{1}, u_{1} \geq \bar{\mu} \varphi_{1}$ and $T u_{1} \geq \lambda_{1} \bar{\mu} L_{1} \varphi_{1}=\bar{\mu} \varphi_{1}$. Thus,

$$
u_{1}=T u_{1}+\mu_{1} \varphi_{1} \geq \bar{\mu} \varphi_{1}+\mu_{1} \varphi_{1}=\left(\bar{\mu}+\mu_{1}\right) \varphi_{1}
$$

which contradicts the definition of $\bar{\mu}$ So (3.6) is true and by lemma 2.4 we have

$$
i\left(T, K_{r_{1}}, K\right)=0 .
$$

On the other hand, by (H3), we have $g_{\infty}<\frac{\lambda 2}{\lambda}$, there exists $0<\sigma<1$ and $r_{2}>r_{1}>0$ such that

$$
g(u) \leq \sigma \frac{\lambda_{2}}{\lambda} u, \quad u \geq r_{2} .
$$

Let $L \phi=\sigma \lambda_{2} L_{2} \phi, \phi \in C[0,1]$. Then $L: C[0,1] \rightarrow C[0,1]$ is a bounded linear operator and $L(K) \subset K$. Denote

$$
M=\left(\max _{0 \leq t, s \leq 1} G^{*}(t, s)\right) \sup _{\varphi \in \partial K_{\mathrm{r}_{2}}} \int_{0}^{1} \lambda a(s) q_{2}(s) g(\varphi(s)) d s .
$$


It is clear that $M<+\infty$ Let

$$
W=\{\varphi \in K \mid \varphi=\mu T \varphi, 0 \leq \mu \leq 1\} .
$$

In the following, we prove that $W$ is bounded.

For any $\phi \in W$, set $\bar{\varphi}(t)=\min \left\{\varphi(t), r_{2}\right\}$ and denote $E(\phi)=\left\{t \in[0,1] \mid \phi(t)>r_{2}\right\}$, then

$$
\begin{aligned}
\varphi(t) & =\mu(T \varphi)(t) \leq(T \varphi)(t)=\lambda \int_{0}^{1} G^{*}(t, s) a(s) f\left(s, s^{\alpha-2} \varphi(s)\right) d s \\
& \leq \lambda \int_{0}^{1} G^{*}(t, s) a(s) q_{2}(s) g(\varphi(s)) d s \\
& =\lambda \int_{E(\varphi)} G^{*}(t, s) a(s) q_{2}(s) g(\varphi(s)) d s+\lambda \int_{[0,1] \backslash E(\varphi)} G^{*}(t, s) a(s) q_{2}(s) g(\bar{\varphi}(s)) d s \\
& \leq \sigma \lambda_{2} \int_{0}^{1} G^{*}(t, s) a(s) q_{2}(s) \varphi(s) d s+\lambda \int_{0}^{1} G^{*}(t, s) a(s) q_{2}(s) g(\bar{\varphi}(s)) d s \\
& \leq(L \varphi)(t)+M, t \in[0,1] .
\end{aligned}
$$

Thus $((I-L) \phi)(t) \leq M, t \in[0,1]$ : Since $\lambda_{2}$ is the first eigenvalue of $L_{2}$ and $0<\sigma<1$, the first eigenvalue of $L,(r(L))^{-1}>1$. Therefore, the inverse operator $(I-L)^{-1}$ exists and

$$
(I-L)^{-1}=I+L+L^{2}+\cdots+L^{n} \cdots
$$

It follows from $L(K) \subset K$ that $(I-L)^{-1}(K) \subset K$. So we have $\phi(t) \leq(I-L)^{-1} M, t \in[0,1]$ and $W$ is bounded.

Choose $r_{3}>\max \left\{r_{2},\left\|(I-L)^{-1} M\right\|\right\}$. Then by lemma 2.5 , we have

$$
i\left(T, K_{r_{3}}, K\right)=1 \text {. }
$$

By (3.7) and (3.8), one has

$$
i\left(T, K_{r_{3}} \backslash \bar{K}_{r_{1}}, K\right)=i\left(T, K_{r_{3}}, K\right)-i\left(T, K_{r_{1}}, K\right)=1 .
$$

Then $T$ has at least one fixed point on $K_{r_{3}} \backslash \bar{K}_{r_{1}}$ By Lemma 3.1, this means that problem (1.6) has at least one positive solution. The proof is complete.

Theorem 3.4 Assume $(\mathrm{H} 1)(\mathrm{H} 2)(\mathrm{H} 4)$ hold. Then the BVP (1.6) has at least one positive solution.

Theorem 3.5 Assume (H1)(H2) hold and $\frac{\lambda_{1}}{\lambda}<g_{0} \leq \infty, \frac{\lambda_{1}}{\lambda}<g_{\infty} \leq \infty$. Moreover, the following condition holds:

(H5) there exists a $p>0$ such that $0 \leq u \leq p$ implies $g(u)<M_{1} p$, where $M_{1}=\left[\lambda \int_{0}^{1} \Phi(s) a(s) q_{2}(s) d s\right]^{-1}$.

Then the BVP (1.6) has at least two positive solution.

Theorem 3.6 Assume $(\mathrm{H} 1)(\mathrm{H} 2)$ hold and $0 \leq g_{0}<\frac{\lambda_{2}}{\lambda}, 0 \leq g_{\infty}<\frac{\lambda_{2}}{\lambda}$. Moreover, the following condition holds:

(H6) there exists a $p>0$ such that $\frac{p(\alpha-1)}{16} \leq u \leq p$ implies $g(u)>M_{2} p$, where $M_{2}=\left[\lambda \int_{\frac{1}{4}}^{\frac{3}{4}} G^{*}\left(\frac{1}{2}, s\right) a(s) q_{1}(s) d s\right]^{-1}$. Then the BVP (1.6) has at least two positive solution. 
Remark 3.1 Theorems 3.3, 3.4 extend and improve Theorem 1.5 in [9], Theorems 3.5 and 3.6 extend and improve Theorems 1.3 and 1.4 in [9], respectively.

\section{Example}

Consider the boundary value problem

$$
\left\{\begin{array}{l}
D_{0+}^{\alpha} u(t)+\lambda \mu\left(u^{a}(t)+u^{b}(t)\right)=0, \quad 0<t<1,0<a<1<b<\frac{1}{2-\alpha}, 1<\alpha<2 \\
u(0)=u(1)=0
\end{array}\right.
$$

Then (3.9) have at least two positive solutions $u_{1}$ and $u_{2}$, for each $0<\mu<\mu^{*}$, where $\mu^{*}$ is some positive constant.

Proof. We will apply Theorem 3.5. To this end we take $a(t) \equiv 1, f(t, u)=\mu\left(u^{a}(t)+u^{b}\right.$ $(t))$, then $f\left(t, t^{\alpha-2} y\right)=\mu\left(t^{a(\alpha-2)} y^{a}(t)+t^{b(\alpha-2) y b}(t)\right)$.

Let $q_{1}(t)=t^{a(\alpha-2)}, q_{2}(t)=t^{b(\alpha-2)}$ and $g(y)=\mu\left(y^{a}+y^{b}\right)$, then $q_{1}(t) g(y) \leq f\left(t, t^{\alpha-2} y\right) \leq q_{2}(t)$ $g(y)$ and $q_{1}, q_{2} \in L^{1}(0,1), g \in C([0,+\infty),[0,+\infty))$. Thus (H1)(H2)is satisfied, and it is to see that $\frac{\lambda_{1}}{\lambda}<g_{0} \leq \infty, \frac{\lambda_{1}}{\lambda}<g_{\infty} \leq \infty$.

Since

$$
\begin{aligned}
\frac{1}{\Gamma(\alpha)} \int_{0}^{1} s(1-s)^{\alpha-1} q_{2}(s) d s & =\frac{1}{\Gamma(\alpha)} \int_{0}^{1} s(1-s)^{\alpha-1} s^{b(\alpha-2)} d s \\
& =\frac{1}{\Gamma(\alpha)} \int_{0}^{1} s^{(2+b(\alpha-2))-1}(1-s)^{\alpha-1} d s \\
& =\frac{1}{\Gamma(\alpha)} \frac{\Gamma(2+b(\alpha-2)) \Gamma(\alpha)}{\Gamma(2+b(\alpha-2)+\alpha)} \\
& =\frac{\Gamma(2+b(\alpha-2))}{\Gamma(2+b(\alpha-2)+\alpha)}
\end{aligned}
$$

then $M_{1}=\left(\lambda \frac{\Gamma(2+b(\alpha-2))}{\Gamma(2+b(\alpha-2)+\alpha)}\right)^{-1}$.

Let

$$
\mu^{*}=F(p)=\sup _{y>0}\left\{F(y): F(\gamma)=M_{1} \frac{\gamma}{y^{a}+\gamma^{b}}\right\}=F\left(\left(\frac{1-a}{b-1}\right)^{\frac{1}{b-a}}\right),
$$

where $p=\left(\frac{1-a}{b-1}\right)^{\frac{1}{b-a}}$.

Then, for $\mu<\mu^{*}$, we have

$$
g(\gamma)=\mu\left(\gamma^{a}+\gamma^{b}\right) \leq \mu\left(p^{a}+p^{b}\right)<M_{1} p, 0 \leq \gamma \leq p,
$$

thus (H5) holds. Therefore, (3.9) have at least two positive solutions $u_{1}$ and $u_{2}$, for each $0<\mu<\mu^{*}$.

\section{Acknowledgements}

The authors are grateful to the anonymous referee for their valuable suggestions. X. Han was supported by the NSFC (No. 11101335), the Gansu Provincial Department of Education Fund (No. 1101-02), and the Project of Science and Technology Bureau of Lanzhou (2011-2-72).

\section{Authors' contributions}

The authors declare that the study was realized in collaboration with the same responsibility. All authors read and approved the final manuscript. 


\section{Competing interests}

The authors declare that they have no competing interests.

Received: 14 December 2011 Accepted: 23 May 2012 Published: 23 May 2012

\section{References}

1. El-sayed, AMA: Nonlinear functional differential equations of arbitrary order. Nonlinear Anal 33, 181-186 (1998). doi:10.1016/S0362-546X(97)00525-7. doi:10.1016/S0362-546X(97)00525-7

2. Kilbas, AA, Marichev, Ol, Samko, SG: Fractional Integral and Derivativws (Theory and Applications). Gordon and Breach, Switzerland. (1993)

3. Kilbas, AA, Trujillo, JJ: Differential equations of fractional order: methods, results and problems II. Appl Anal 81, 435-493 (2002). doi:10.1080/0003681021000022032. doi:10.1080/0003681021000022032

4. Kilbas, AA, Trujillo, JJ: Differential equations of fractional order: methods, results and problems I. Appl Anal 78, 153-192 (2001). doi:10.1080/00036810108840931. doi:10.1080/00036810108840931

5. Miller, KS, Ross, B: An Introduction to the Fractional Calculus and Fractional Differential Equations. Wiley, New York (1993)

6. Podlubny, I: Fractional Differential Equations, Mathematics in science and Engineering. Academic Press, New York. (1999)

7. Bai, ZB, Lu, HS: Positive solutions for boundary value problem of nonlinear fractional differential equation. J Math Anal Appl 311, 495-505 (2005). doi:10.1016/j.jmaa.2005.02.052. doi:10.1016/j.jmaa.2005.02.052

8. Bai, ZB: On positive solutions of 495a nonlocal fractional boundary value problem. Non-linear Anal 72, 916-924 (2010). doi:10.1016/j.na.2009.07.033

9. Jiang, $D Q$, Yuan, $C Y$ : The positive properties of the Green function for Dirichlet-type boundary value problems of nonlinear fractional differential equations and its application. Nonlinear Anal 72, 710-719 (2010). doi:10.1016/j. na.2009.07.012. doi:10.1016/j.na.2009.07.012

10. Wang, YQ, Liu, LS, Wu, YH: Positive solutions for a nonlocal fractional differential equation. Nonlinear Anal 74 3599-3605 (2011). doi:10.1016/.na.2011.02.043. doi:10.1016/j.na.2011.02.043

11. Wang, YQ, Liu, LS, Wu, YH: Positive solutions for a class of fractional boundary value problem with changing sign nonlinearity. Nonlinear Anal 74, 6434-6441 (2011). doi:10.1016/j.na.2011.06.026. doi:10.1016/j.na.2011.06.026

12. Zhang, SQ: The existence of a positive solution for a nonlinear fractional differential equation. J Math Anal Appl 252, 804-812 (2000). doi:10.1006/jmaa.2000.7123. doi:10.1006/jmaa.2000.7123

13. Zhang, SQ: Existence of positive solution for some class of nonlinear fractional differential equations. J Math Anal Appl 278, 136-148 (2003). doi:10.1016/50022-247X(02)00583-8. doi:10.1016/S0022-247X(02)00583-8

14. Liang, SH, Zhang, JH: Positive solutions for boundary value problems of nonlinear fractional differential equation. Nonlinear Anal 71, 5545-5550 (2009). doi:10.1016/.jna.2009.04.045. doi:10.1016/..na.2009.04.045

15. Zhao, YG, Sun, SR, Han, ZL, Li, QP: The existence of multiple positive solutions for boundary value problems of nonlinear fractional differential equations. Commun N S N Simulat. 16, 2086-2097 (2011)

16. Zhao, YG, Sun, SR, Han, ZL, Zhang, M: Positive solutions for boundary value problems of nonlinear fractional differential equations. Appl Math Comput 217, 6950-6958 (2011). doi:10.1016/j.amc.2011.01.103. doi:10.1016/j.amc.2011.01.103

17. Yang, $\mathrm{X}$, Wei, ZL, Dong, W: Existence of positive solutions for the boundary value problems of nonlinear fractional differential equations. Commun N S N Simulat. 17, 85-92 (2012)

18. Henderson, J, Wang, HY: Positive solutions for nonlinear eigenvalue problems. J Math Anal Appl. 208, 252-259 (1997). doi:10.1006/jmaa.1997.5334

19. Chang, YK, Kavitha, V, Arjunan, MM: Existence and uniqueness of mild solutions to a semilinear integrodifferential equation of fractional order. Nonlinear Anal 71, 5551-5559 (2009). doi:10.1016/j.na.2009.04.058. doi:10.1016/j. na.2009.04.058

20. Chang, YK, Li, WS: Solvability for impulsive neutral integro-differential equations with state-dependent delay via fractional operators. J Optim Theory Appl. 144, 445-459 (2010). doi:10.1007/s10957-009-9612-6

21. Chang, YK, Arjunan, MM, Kavitha, V: Existence results for neutral functional integrodifferential equations with infinite delay via fractional operators. J Appl Math Comput 36, 201-218 (2011). doi:10.1007/s12190-010-0397-4. doi:10.1007/ s12190-010-0397-4

22. Gao, HL, Han, XL: Existence of positive solutions for fractional differential equation with nonlocal boundary condition. Inter Diff Equ 2011 (2011). Art ID 328394. doi:10.1155/2011/328394

doi:10.1186/1687-1847-2012-66

Cite this article as: Han and Gao: Existence of positive solutions for eigenvalue problem of nonlinear fractional differential equations. Advances in Difference Equations 2012 2012:66. 